\title{
PASSMAN-ZALESSKII RADICAL OF GROUP ALGEBRAS
}

\author{
by I. SINHA \\ (Received 12th March 1973)
}

Recently Passman (attributing the origin of the idea to Zalesskii) has defined the following ideal in a ring, (2).

Definition. For a unitary ring $R$,

$N^{*} R=\{\alpha \in R \mid \alpha S$ is nilpotent for all finitely generated subrings $S$ of $R\}$.

For a group algebra $K G$ over a field $K$ of characteristic $p \neq 0$, he has proved the radical property:

$$
N^{*}\left(K(G) / N^{*} K(G)\right)=0
$$

We shall therefore call $N^{*} K(G)$, the Passman-Zalesskii Radical (PZ-Radical, in short) of $K G$.

If one defines

$$
\begin{aligned}
& \wedge(G)=\left\{g \in G|| S: S \cap C_{G}(g) \mid<\infty \text { for all finitely generated subgroups } S\right. \\
& \quad \text { of } G\}, \\
& \wedge^{+}(G)=\{g \in \wedge(G)|| g \mid<\infty\}, \text { and } \\
& \left.\wedge^{p}(G)=\left\langle g \in \wedge^{+}(G)|| g\right|=p^{e} \text { for some } e\right\rangle,
\end{aligned}
$$

then Passman has proved that,

$$
N^{*} K(G)=J K \wedge^{+}(G) \cdot K G
$$

where $J$ denotes the Jacobson Radical, (2).

Here we want to prove:

Theorem 1. $N^{*} K(G)=J K \wedge^{p}(G) . K G$.

This will be proved, if we can prove:

Theorem 2. $J K \wedge^{+}(G)=J K \wedge^{p}(G) \cdot K \wedge^{+}(G)$.

We firstly state the following result proved by Passman (2):

Lemma 1. Let $W \unlhd G, W \subset \wedge^{+}(G)$. Then,

$$
J K(W) . K G \subseteq N^{*} K(G) \subseteq J K(G) .
$$

If we put $G=\wedge^{+}(G), W=\wedge^{p}(G)$ in Lemma 1, we obtain,

Lemma 2. $J K\left(\wedge^{p}(G)\right) \subseteq J K\left(\wedge^{+}(G)\right)$.

We shall also note the following result, though we shall not need it here: 
Proposition. $J K\left(\wedge^{+}(G)\right) \subseteq \mathfrak{A}\left(\wedge^{P}(G)\right)$, where $\mathfrak{A}\left(\wedge^{p}(G)\right)$ is the augmentation ideal of $\wedge^{p}(G)$ in $\wedge^{+}(G)$, (3).

Proof. $\wedge^{+}(G) / \wedge^{p}(G)$ is a locally finite group whose elements have order prime to $p,(2)$. Therefore by Theorem 18.7 of $(1), K\left(\wedge^{+}(G) / \wedge^{p}(G)\right)$ is semisimple. But this algebra is isomorphic to $K\left(\wedge^{+}(G)\right) / \mathfrak{A}\left(\wedge^{p}(G)\right)$. Hence the result follows.

Proof of Theorem 2. In view of Lemma 2 above, we merely have to prove that $J K \wedge^{+}(G) \subseteq J K \wedge^{p}(G) \cdot K \wedge^{+}(G)$. Let $a \in J K \wedge^{+}(G)$. Then $T=\langle$ Supp $a\rangle$ is a finite subroup of $\wedge^{+}(G)$, since the latter is locally finite. Hence

$$
H=T \cdot \wedge^{p}(G) \supseteq \operatorname{Supp} a,\left|H: \wedge^{p}(G)\right|<\infty \text { and } p|| H: \wedge^{p}(G) \mid
$$

since $\wedge^{+}(G) / \wedge^{p}(G)$ is a locally finite group with no elements of order divisible by $p$. Then by Theorem 16.6 of (1),

$$
J K H=J K \wedge^{p}(G) . K H .
$$

Now $a \in J K \wedge^{+}(G) \cap K H \subseteq J K H$ by Lemma 16.9 of (1). Hence

This proves the theorem.

$$
a \in J K \wedge^{p}(G) \cdot K \wedge^{+}(G)
$$

Alternatively the fact that

$$
J K \wedge^{+}(G) \subseteq J K \wedge^{p}(G) \cdot K \wedge^{+}(G)
$$

is also an immediate consequence of the corollary on page 55 of (4), and the fact that $\wedge^{+}(G) / \wedge^{p}(G)$ is locally finite with no element of order divisible by p. (The author wishes to thank the referee for pointing out the reference (4).)

Corollary. $\wedge^{p}(G)=1$ implies that

(i) $K \wedge^{+}(G)$ is semi-simple and (ii) the PZ-Radical of $K(G)=0$.

This compares with Theorem 18.7 of (1).

\section{REFERENCES}

(1) D. S. Passman, Infinite Group-Rings (Marcel Dekker Inc., New York, 1971).

(2) D. S. Passman, A New Radical for Group-Rings (to appear).

(3) I. SinHA, Augmentation-maps of Subgroups of a group, Math. Z. 94 (1966), 193-206.

(4) D. A. R. WAllace, Some Applications of Subnormality in groups in the study of Group-Algebras, Math. Z. 108 (1968), 53-62.

Michigan State University

East Lansing, Michigan 48823 